\title{
A NOTE ON SIMULTANEOUS CONGRUENCES, II: MORDELL REVISED
}

\author{
TREVOR D. WOOLEY
}

(Received 15 September 2009; accepted 25 January 2010)

\author{
Communicated by I. E. Shparlinski
}

\begin{abstract}
When $p$ is a prime number, and $k_{1}, \ldots, k_{t}$ are natural numbers with $1 \leq k_{1}<k_{2}<\cdots<k_{t}<p$, we show that the simultaneous congruences $\sum_{1}^{t} x_{i}^{k_{j}} \equiv \sum_{1}^{t} y_{i}^{k_{j}} \bmod p(1 \leq j \leq t)$ possess at most $k_{1} \cdots k_{t} p^{t}$ solutions with $1 \leq x_{i}, y_{i} \leq p(1 \leq i \leq t)$. Analogous conclusions are provided when one or more of the exponents $k_{i}$ is negative.
\end{abstract}

2000 Mathematics subject classification: primary 11D79; secondary 11L15.

Keywords and phrases: congruences in many variables, exponential sums.

\section{Introduction}

In a paper devoted to generalizations of Gauss sums published in 1932, Mordell [11] obtained upper bounds for exponential sums over finite fields through estimates for their mean values. Although eclipsed by Weil's resolution of the Riemann hypothesis for curves over finite fields (see [13]), Mordell's approach motivates Vinogradov's use [12] of mean values in estimating Weyl sums and their generalizations, and as such leaves an indelible mark on the literature. Weil's estimates are worse than trivial when the degree of the exponential sum is large compared to the associated prime modulus, and in recent years much effort has been expended on deriving estimates that remain nontrivial for larger degrees (see [1, 2, 4-7, 10, 16]). The bulk of this work revisits Mordell's original approach, and is based on an estimate for the number of solutions of certain polynomial congruences equivalent to a mean value estimate (see [11, Equation (16)]). Mordell's proof of this estimate involves notions of independent parameters and unstated elements of elimination theory that are vestiges of a bygone era prior to the development of modern algebraic geometry. As such, the mathematical reader of today will likely demand some renovation of this proof going beyond a lick

The author is supported by a Royal Society Wolfson Research Merit Award.

(C) 2010 Australian Mathematical Publishing Association Inc. 1446-7887/2010 \$16.00 
of paint to a certain amount of structural reinforcement. Our object in this note is to address the latter concerns, at the same time providing an estimate sharper than that due to Mordell that is, in many respects, best possible. Improved estimates for certain associated exponential sums are immediate corollaries of our sharper bounds.

Before announcing our conclusions, we must introduce some notation. When $q$ is a power of a prime number $p$, we denote by $\mathbb{F}_{q}$ the finite field having $q$ elements. Let $t$ be a natural number, and suppose that $k_{1}, \ldots, k_{t}$ are positive integers. We write $N_{t}(q ; \mathbf{k})$ for the number of solutions of the system of equations

$$
x_{1}^{k_{j}}+\cdots+x_{t}^{k_{j}}=x_{t+1}^{k_{j}}+\cdots+x_{2 t}^{k_{j}} \quad(1 \leq j \leq t)
$$

with $\mathbf{x} \in \mathbb{F}_{q}^{2 t}$. In view of the relation $a^{q}=a$, valid for each element $a$ of $\mathbb{F}_{q}$, it is apparent that there is no loss of generality in supposing that

$$
1 \leq k_{1}<k_{2}<\cdots<k_{t}<q \text {. }
$$

Furthermore, since the characteristic of $\mathbb{F}_{q}$ is $p$, for each natural number $l$ one has

$$
\left(x_{1}^{l}+x_{2}^{l}+\cdots+x_{t}^{l}\right)^{p}=x_{1}^{l p}+x_{2}^{l p}+\cdots+x_{t}^{l p} .
$$

Thus we see that there is in addition no loss of generality in restricting attention to systems (1.1) for which $p \nmid k_{j}(1 \leq j \leq t)$.

THEOREM 1.1. Suppose that $k_{1}, \ldots, k_{t}$ are integers with $p \nmid k_{j}(1 \leq j \leq t)$ and satisfying the condition (1.2). Then

$$
N_{t}(q ; \mathbf{k}) \leq k_{1} k_{2} \cdots k_{t} q^{t} .
$$

In the situation in which $q$ is a prime number, the estimate (16) of Mordell [11] supplies the bound

$$
N_{t}(q ; \mathbf{k}) \leq \frac{(2 t) !}{(t !)^{2}} k_{1} k_{2} \cdots k_{t} q^{t}
$$

Under the same circumstances, Cochrane and Pinner (see [6, Lemma 3.1]) provide an estimate analogous to this upper bound of Mordell, save that the right-hand side is multiplied by a factor $4 / t^{2}$. Their proof avoids Mordell's result, instead applying a version of Bézout's theorem for nonsingular solutions of polynomial congruences due to the author [15], and as such provides a robust proof of Mordell's estimate. The conclusion of Theorem 1.1 is superior to both the estimates of Mordell and of Cochrane and Pinner. Moreover, as is apparent from the discussion of [6, Example 3.1], when $k$ is a fixed natural number with $k \mid(q-1)$, and $k_{i}=i k(1 \leq i \leq t)$, one has the lower bound

$$
N_{t}(q ; \mathbf{k}) \geq k_{1} k_{2} \cdots k_{t}\left(q^{t}+O_{t}\left(q^{t-1}\right)\right),
$$

and thus inequality (1.3) is asymptotically sharp. In some sense, therefore, the conclusion of Theorem 1.1 is best possible. We should note in this context that in the 
special case in which $t=2$ and $q$ is a prime number, the conclusion of Theorem 1.1 is derived in [14, Lemma 7], and is also recorded rather later in [6, Lemma 3.2].

Consider next the situation analogous to that described in the preamble to Theorem 1.1 in which the exponents $k_{1}, \ldots, k_{t}$ are now nonzero integers, but potentially not all of the same sign. In this situation, we define $M_{t}(q ; \mathbf{k})$ to be the number of solutions of the system of equations (1.1) with $\mathbf{x} \in\left(\mathbb{F}_{q}^{\times}\right)^{2 t}$. Here, we may suppose without loss of generality that

$$
-q<k_{1}<k_{2}<\cdots<k_{t}<q,
$$

that $p \nmid k_{j}(1 \leq j \leq t)$, and further that

$$
(q-1) \nmid\left(k_{i}-k_{j}\right) \quad(1 \leq i<j \leq t) .
$$

Finally, it is convenient to put

$$
l_{i}= \begin{cases}k_{i} & \text { when } k_{i}>0 \\ t\left|k_{i}\right| & \text { when } k_{i}<0\end{cases}
$$

and

$$
m_{i}= \begin{cases}k_{i} & \text { when } k_{i}>0, \\ (2 t-1)\left|k_{i}\right| & \text { when } k_{i}<0 .\end{cases}
$$

THEOREM 1.2. Suppose that $k_{1}, \ldots, k_{t}$ are integers with $p \nmid k_{j}(1 \leq j \leq t)$ and satisfying conditions (1.4) and (1.5). Then one has the estimates

$$
\begin{aligned}
& M_{t}(q ; \mathbf{k}) \leq m_{1} m_{2} \cdots m_{t}(q-1)^{t}, \\
& M_{t}(q ; \mathbf{k}) \leq 2^{2 t}\left|k_{1} k_{2} \cdots k_{t}\right|(q-1)^{t}, \\
& M_{t}(q ; \mathbf{k}) \leq(t+1) l_{1} l_{2} \cdots l_{t}(q-1)^{t} .
\end{aligned}
$$

By way of comparison, when $q$ is a prime number, estimate (16) of Mordell [11] essentially provides the bound

$$
M_{t}(q ; \mathbf{k}) \leq \frac{(2 t) !}{(t !)^{2}} l_{1} \cdots l_{t}(q-1)^{t}
$$

One may verify that the third estimate of Theorem 1.2 is superior to (1.6) for every natural number $t$ exceeding 1. Moreover, the second estimate of Theorem 1.2 will be superior to the third whenever $t>2$, and the number of the exponents $k_{i}$ that are negative is more than about $(2 \log 2) t / \log t$. We should also remark that Lemma 3.2 of Cochrane and Pinner [6] establishes an estimate matching the first provided by Theorem 1.2 in those special cases in which $t=2$ and $q$ is prime.

We finish by recording some consequences of Theorems 1.1 and 1.2 for exponential sums. Let $\chi$ be a Dirichlet character modulo $p$, and write $e_{p}(z)$ for $e^{2 \pi i z / p}$. 
Then, when $\mathbf{a} \in\left(\mathbb{F}_{p}^{\times}\right)^{t}$, we define the Laurent polynomial $f(x)=a_{1} x^{k_{1}}+\cdots+a_{t} x^{k_{t}}$, and also the mixed exponential sum

$$
S(\chi, f)=\sum_{x \in \mathbb{F}_{p}^{\times}} \chi(x) e_{p}(f(x)) .
$$

COROLlary 1.3. When $k_{1}, \ldots k_{t}$ are nonzero integers with $p \nmid k_{j}(1 \leq j \leq t)$ and satisfying $1 \leq k_{1}<k_{2}<\cdots<k_{t}<p$, then

$$
|S(\chi, f)| \leq\left(k_{1} \cdots k_{t}\right)^{1 / t^{2}} p^{1-1 /(2 t)} .
$$

When instead $-p<k_{1}<k_{2}<\cdots<k_{t}<p$ and $p \nmid\left(k_{i}-k_{j}\right)$ for $1 \leq i<j \leq t$, then one has the estimates

$$
\begin{aligned}
& |S(\chi, f)| \leq\left(m_{1} m_{2} \cdots m_{t}\right)^{1 / t^{2}} p^{1-1 /(2 t)}, \\
& |S(\chi, f)| \leq 2^{2 / t}\left|k_{1} \cdots k_{t}\right|^{1 / t^{2}} p^{1-1 /(2 t)}, \\
& |S(\chi, f)| \leq(t+1)^{1 / t^{2}}\left(l_{1} \cdots l_{t}\right)^{1 / t^{2}} p^{1-1 /(2 t)} .
\end{aligned}
$$

For comparison, Theorem 1.1 of Cochrane and Pinner [6] provides the bound

$$
|S(\chi, f)| \leq 2^{2 / t}\left(l_{1} \cdots l_{t}\right)^{1 / t^{2}} p^{1-1 /(2 t)} .
$$

This is weaker than estimate (1.9) in all cases, since $t+1<4^{t}$ for every positive integer $t$. It is also weaker than (1.8) whenever there is a change of sign amongst the $k_{i}$. Finally, in circumstances where the $k_{i}$ are all of the same sign, estimate (1.7) yields bounds superior to those of $(1.10)$ by a factor $2^{2 / t}$. See $[4,5]$ for refinements in special cases that may prove superior to the estimates of Corollary 1.3.

Although the conclusion of Theorem 1.1 is, in some sense, best possible, it is not apparent what the truth may be for the analogous situation examined in Theorem 1.2, in which the exponents are of mixed sign. Let $w$ be a natural number, put $t=2 w$, and write

$$
\mathbf{a}=(-w, 1-w, \ldots,-2,-1,1,2, \ldots, w-1, w) .
$$

Then by considering solutions $\mathbf{x}$ of (1.1) in which $\left(x_{1}^{k}, \ldots, x_{2 w}^{k}\right)$ is a permutation of $\left(x_{2 w+1}^{k}, \ldots, x_{4 w}^{k}\right)$, one finds that, when $q$ is large and $k \mid(q-1)$,

$$
M_{2 w}(q ; k \mathbf{a}) \geq(2 w) ! k^{2 w}(q-1)^{2 w}+O_{k, w}\left(q^{2 w-1}\right) .
$$

Consequently, the bound

$$
M_{t}(q ; \mathbf{k}) \leq C_{t}\left|k_{1} \cdots k_{t}\right|(q-1)^{t}
$$

cannot hold in general when $C_{t}<\left(\begin{array}{c}t \\ {[t / 2]}\end{array}\right)$. 


\section{Counting solutions of equations in finite fields}

In this section we bound $N_{t}(q ; \mathbf{k})$ and $M_{t}(q ; \mathbf{k})$ by making use of rough data available from modern versions of Bézout's theorem, in combination with crude but robust estimates for the number of $\mathbb{F}_{q}$-rational points on algebraic varieties made available only relatively recently. We begin with a direct consequence of Bézout's theorem. In this context, we write $\operatorname{deg}(W)$ for the degree of a variety $W$, and $\overline{\mathbb{F}}_{q}$ for the algebraic closure of $\mathbb{F}_{q}$.

Lemma 2.1. Suppose that $f_{i}(\mathbf{x}) \in \overline{\mathbb{F}}_{q}\left[x_{1}, \ldots, x_{s}\right]$ is a polynomial of degree $d_{i}$ for $1 \leq i \leq t$. Let $V_{1}, \ldots, V_{h} \subset \mathbb{A}^{s}$ be the components of the complete intersection defined by the system of equations $f_{i}(\mathbf{x})=0(1 \leq i \leq t)$. Then

$$
\sum_{i=1}^{h} \operatorname{deg}\left(V_{i}\right) \leq d_{1} d_{2} \cdots d_{t} .
$$

PROOF. Let $Y$ be a variety in $\mathbb{P}^{n}$, and let $H$ be a hypersurface not containing $Y$. Also, let $Z_{1}, \ldots, Z_{m}$ be the irreducible components of $Y \cap H$, and let $i\left(Y, H ; Z_{j}\right)$ denote the intersection multiplicity of the varieties $Y$ and $H$ along $Z_{j}$. Then according to Theorem 7.7 of Hartshorne [8, Ch. 1],

$$
\sum_{j=1}^{m} i\left(Y, H ; Z_{j}\right) \operatorname{deg} Z_{j}=(\operatorname{deg} Y)(\operatorname{deg} H) .
$$

But $i\left(Y, H ; Z_{j}\right) \geq 1$ for each $j$, and so it follows by induction that for the complete intersection defined by the $t$ polynomials in question,

$$
\sum_{i=1}^{h} \operatorname{deg}\left(V_{i}\right) \leq \prod_{i=1}^{t} \operatorname{deg}\left(f_{i}\right)=d_{1} d_{2} \cdots d_{t} .
$$

This concludes the proof.

Next we provide an upper bound for the number of $\mathbb{F}_{q}$-rational points on a variety of given degree and dimension.

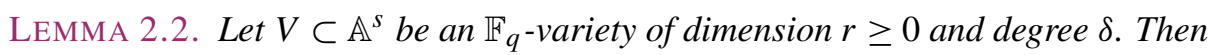

$$
\operatorname{card}\left(V \cap \mathbb{F}_{q}^{s}\right) \leq \delta q^{r}
$$

and

$$
\operatorname{card}\left(V \cap\left(\mathbb{F}_{q}^{\times}\right)^{s}\right) \leq \delta(q-1)^{r} .
$$

PRoOF. The first estimate is supplied by Lemma 2.1 of Cafure and Matera [3]. The second estimate may be established using an immediate modification of the argument of the proof of the latter lemma. For when $1 \leq i \leq s$, we may take $W_{i} \subset \mathbb{A}^{s}$ to be the $\mathbb{F}_{q}$-hypersurface defined by $x_{i}^{q-1}-1$. Then $V \cap\left(\mathbb{F}_{q}^{\times}\right)^{s}=V \cap W_{1} \cap \cdots \cap W_{s}$, 
and so from the argument underlying the proof of Proposition 2.3 of [9], we obtain the inequality

$$
\operatorname{card}\left(V \cap\left(\mathbb{F}_{q}^{\times}\right)^{s}\right) \leq \operatorname{deg}\left(V \cap W_{1} \cap \cdots \cap W_{s}\right) \leq \delta(q-1)^{r} .
$$

This completes the proof of the lemma.

By combining Lemmas 2.1 and 2.2, we obtain an estimate for the number of $\mathbb{F}_{q}$-rational points on a complete intersection.

Lemma 2.3. Suppose that $f_{i}(\mathbf{x}) \in \overline{\mathbb{F}}_{q}\left[x_{1}, \ldots, x_{s}\right]$ is a polynomial of degree $d_{i}$ for $1 \leq i \leq t$. Let $V_{1}, \ldots, V_{n}$ be the components in $\overline{\mathbb{F}}_{q}^{s}$ of the complete intersection $V$ defined by the system of equations $f_{i}(\mathbf{x})=0(1 \leq i \leq t)$, and denote by $U_{r}$ the union of the components $V_{1}, \ldots, V_{n}$ having dimension not exceeding $r$. Then

$$
\operatorname{card}\left(U_{r} \cap \mathbb{F}_{q}^{s}\right) \leq d_{1} d_{2} \cdots d_{t} q^{r} .
$$

In the analogous situation in which we consider the complete intersection in $\left(\overline{\mathbb{F}}_{q}^{\times}\right)^{s}$, one has instead

$$
\operatorname{card}\left(U_{r} \cap\left(\mathbb{F}_{q}^{\times}\right)^{s}\right) \leq d_{1} d_{2} \cdots d_{t}(q-1)^{r} .
$$

PROOF. Suppose that $V_{i_{1}}, \ldots, V_{i_{l}}$ are the components of $V$ having dimension not exceeding $r$. Then $U_{r}$ is the union of $V_{i_{1}}, \ldots, V_{i_{l}}$, so by first applying Lemma 2.2, and then Lemma 2.1, we obtain

$$
\operatorname{card}\left(U_{r} \cap \mathbb{F}_{q}^{s}\right) \leq \sum_{j=1}^{l} \operatorname{deg}\left(V_{i_{j}}\right) q^{r} \leq q^{r} \sum_{i=1}^{n} \operatorname{deg}\left(V_{i}\right) \leq d_{1} d_{2} \cdots d_{t} q^{r} .
$$

The second conclusion of the lemma follows in like manner.

In order to discuss the singular locus of the complete intersection (1.1), we require a lemma concerning the rank of matrices of Vandermonde type. Suppose that $k_{1}, \ldots, k_{t}$ are distinct integers, and that $s$ is a natural number with $s \geq t$. When $i_{1}, \ldots, i_{t}$ are natural numbers with $1 \leq i_{1}<i_{2}<\cdots<i_{t} \leq s$, we define the determinant

$$
\Delta_{\mathbf{i}}(\mathbf{x} ; \mathbf{k})=\operatorname{det}\left(x_{i_{l}}^{k_{j}-1}\right)_{1 \leq j, l \leq t} .
$$

Also, when $k_{i}>0(1 \leq i \leq t)$, we define $\mathfrak{X}_{s}(\mathbf{k})$ to be the set of points $\mathbf{x} \in \overline{\mathbb{F}}_{q}^{s}$ satisfying the system of equations

$$
\Delta_{\mathbf{i}}(\mathbf{x} ; \mathbf{k})=0 \quad\left(1 \leq i_{1}<i_{2}<\cdots<i_{t} \leq s\right) .
$$

Likewise, without conditions on $\mathbf{k}$, we define $\mathfrak{Y}_{s}(\mathbf{k})$ to be the set of points $\mathbf{x} \in\left(\overline{\mathbb{F}}_{q}^{\times}\right)^{s}$ satisfying the system of equations (2.1).

LEMMA 2.4.

(i) Suppose that $k_{1}, \ldots, k_{t}$ are integers with $1 \leq k_{1}<\cdots<k_{t}<q$, and that $s \geq t$. Then the components of the complete intersection $\mathfrak{X}_{s}(\mathbf{k})$ have dimension at most $t-1$. 
(ii) Suppose that $k_{1}, \ldots, k_{t}$ are integers with $-q<k_{1}<\cdots<k_{t}<q$, and further that $(q-1) \mid\left(k_{i}-k_{j}\right)$ for no indices $i$ and $j$ with $1 \leq i<j \leq t$. Then for $s \geq t$, the components of the complete intersection $\mathfrak{Y}_{s}(\mathbf{k})$ have dimension at most $t-1$.

PROOF. The proof of part (ii) of the lemma may be applied, mutatis mutandis, to establish part (i); all that is required is to include 0 as a possible value of each coordinate. We therefore consider only part (ii), and assume the hypotheses ambient in that part of the lemma.

Consider the subset $\mathfrak{Y}_{s}(\mathbf{k}) \subset\left(\overline{\mathbb{F}}_{q}^{\times}\right)^{s}$ defined by the vanishing of all the $(t \times t)$ determinants (2.1). The elements $\mathbf{x}$ of $\mathfrak{Y}_{s}(\mathbf{k})$ may be classified according to the dimension of the linear space spanned by the column vectors $\left(x_{i}^{k_{j}-1}\right)_{1 \leq j \leq t}$ for $1 \leq i \leq s$. This space must have affine dimension at most $t-1$ for every element $\mathbf{x}$ of $\mathfrak{Y}_{s}(\mathbf{k})$, for if the dimension were larger, then one could find a nonvanishing $(t \times t)$-determinant $\Delta_{\mathbf{i}}(\mathbf{x} ; \mathbf{k})$, contradicting the definition of $\mathfrak{Y}_{s}(\mathbf{k})$.

Let $m$ be an integer with $1 \leq m \leq t-1$, consider indices $i_{l}(1 \leq l \leq m)$ with

$$
1 \leq i_{1}<i_{2}<\cdots<i_{m} \leq s,
$$

and suppose that the column vectors $\left(x_{i_{l}}^{k_{j}-1}\right)_{1 \leq j \leq t}$ are linearly independent for $1 \leq$ $l \leq m$. Write $\mathfrak{T}_{m}(\mathbf{i})$ for the set of points $\mathbf{x} \in \mathfrak{Y}_{s}(\mathbf{k})$ satisfying the property that for $1 \leq i \leq s$, all of the column vectors $\left(x_{i}^{k_{j}-1}\right)_{1 \leq j \leq t}$ belong to the linear space spanned by vectors of the above type, and let $\mathfrak{Y}_{s}^{(m)}(\mathbf{k})$ denote the union of the sets $\mathfrak{T}_{m}(\mathbf{i})$ over all choices of $\mathbf{i}$ satisfying (2.2). Then one finds that $\mathfrak{Y}_{s}(\mathbf{k})$ is the union of $\mathfrak{Y}_{s}^{(1)}(\mathbf{k}), \mathfrak{Y}_{s}^{(2)}(\mathbf{k}), \ldots, \mathfrak{Y}_{s}^{(t-1)}(\mathbf{k})$. Moreover, for $1 \leq m \leq t-1$, the set $\mathfrak{T}_{m}(\mathbf{i})$ is determined by the nonvanishing of at least one $(m \times m)$-determinant involving the variables $x_{i_{1}}, \ldots, x_{i_{m}}$, together with the vanishing of all $((m+1) \times(m+1))$ determinants obtained by adjoining another variable $x_{i}$ with $i \notin\left\{i_{1}, \ldots, i_{m}\right\}$. The determinants in question here are of submatrices of the matrix

$$
\left(x_{i}^{k_{j}-1}\right)_{\substack{1 \leq j \leq t \\ 1 \leq i \leq s}}
$$

It follows that each $x_{i}$ with $i \notin\left\{i_{1}, \ldots, i_{m}\right\}$ satisfies a nontrivial polynomial equation determined by $x_{i_{1}}, \ldots, x_{i_{m}}$. We therefore deduce that the components of $\mathfrak{Y}_{s}^{(m)}(\mathbf{k})$ have affine dimension at most $m$, whence the components of $\mathfrak{Y}_{s}(\mathbf{k})$ have affine dimension at most $t-1$. This completes the proof of the lemma.

We are now equipped to establish the principal conclusions of this note.

PROOF OF THEOREM 1.1. Suppose that $k_{1}, \ldots, k_{t}$ are natural numbers such that $1 \leq k_{1}<k_{2}<\cdots<k_{t}$. Recall from the discussion in the preamble to the statement of Theorem 1.1 that we may suppose, without loss of generality, that $k_{t}<q$ and $p \nmid k_{i}(1 \leq i \leq t)$. Consider the complete intersection $\mathcal{Z}$ defined by the simultaneous equations (1.1) with $\mathbf{x} \in \overline{\mathbb{F}}_{q}^{2 t}$. Note that $\mathcal{Z}$ is defined by a system of $t$ polynomial 
equations, of respective degrees $k_{1}, \ldots, k_{t}$, in $2 t$ variables. Let $\mathcal{Z}_{1}, \ldots, \mathcal{Z}_{d}$ be the distinct components of $\mathcal{Z}$. We claim that the affine dimension of each component $\mathcal{Z}_{i}$ is at most $t$. If such were not the case for the component $\mathcal{Z}_{i}$, then the intersection (1.1) would be improper, and $\mathcal{Z}_{i}$ would belong to the singular locus of $\mathcal{Z}$. The latter is contained within the set of points $\mathbf{x} \in \overline{\mathbb{F}}_{q}^{2 t}$ satisfying the simultaneous equations

$$
\operatorname{det}\left(k_{j} x_{i_{l}}^{k_{j}-1}\right)_{1 \leq j, l \leq t}=0,
$$

with $1 \leq i_{1}<i_{2}<\cdots<i_{t} \leq 2 t$. Since $p \nmid k_{j}(1 \leq j \leq t)$, it follows that this singular locus is contained in the set $\mathfrak{X}_{2 t}(\mathbf{k})$ defined in the preamble to Lemma 2.4. It therefore follows from Lemma 2.4(i) that the component $\mathcal{Z}_{i}$ in question must have dimension at most $t-1$, contradicting our earlier hypothesis.

We have shown that the components $\mathcal{Z}_{1}, \ldots, \mathcal{Z}_{d}$ of $\mathcal{Z}$ each have dimension at most $t$, and so we may infer from Lemma 2.3 that

$$
N_{t}(q ; \mathbf{k})=\operatorname{card}\left(\mathcal{Z} \cap \mathbb{F}_{q}^{2 t}\right) \leq k_{1} k_{2} \cdots k_{t} q^{t} .
$$

This completes the proof of Theorem 1.1.

Proof OF TheOREM 1.2. Suppose now that $k_{1}, \ldots, k_{t}$ are nonzero integers with $-q<k_{1}<k_{2}<\cdots<k_{t}<q$ for which $(q-1) \nmid\left(k_{i}-k_{j}\right)$ for $1 \leq i<j \leq t$. There is again no loss of generality in supposing that $p \nmid k_{i}(1 \leq i \leq t)$. We suppose that $k_{i}<0$ for $1 \leq i \leq u$ and $k_{i}>0$ for $u+1 \leq i \leq t$. Here, there is no loss of generality in supposing that $u \geq 1$ and $t>u$, for otherwise the conclusion of Theorem 1.1 delivers the desired estimate, if necessary by replacing $x_{i}$ by $x_{i}^{-1}$ for $1 \leq i \leq 2 t$. It is convenient for the purpose of concision to introduce the notational device of writing

$$
\check{x}_{i}=\prod_{\substack{1 \leq l \leq 2 t \\ l \neq i}} x_{l} \quad(1 \leq i \leq 2 t) .
$$

In addition, we write $\kappa_{j}$ for $-k_{j}$. Then by clearing denominators, it is apparent that $M_{t}(q ; \mathbf{k})$ counts the number of solutions of the system

$$
\begin{aligned}
& \sum_{i=1}^{t} \check{x}_{i}^{\kappa_{j}}=\sum_{i=t+1}^{2 t} \check{x}_{i}^{\kappa_{j}} \quad(1 \leq j \leq u), \\
& \sum_{i=1}^{t} x_{i}^{k_{j}}=\sum_{i=t+1}^{2 t} x_{i}^{k_{j}} \quad(u+1 \leq j \leq t),
\end{aligned}
$$

with $\mathbf{x} \in\left(\mathbb{F}_{q}^{\times}\right)^{2 t}$. Notice here that, in view of the definition of $\check{x}_{i}$, the degree of the $j$ th equation in (2.3) is $(2 t-1)\left|k_{j}\right|=m_{j}$.

The system (2.3), (2.4) is defined by a system of $t$ polynomial equations, of respective degrees $m_{1}, \ldots, m_{t}$, in $2 t$ variables. Let $\mathcal{Z}$ be the complete intersection 
defined by the system (2.3), (2.4) with $\mathbf{x} \in\left(\overline{\mathbb{F}}_{q}^{\times}\right)^{2 t}$, and let $\mathcal{Z}_{1}, \ldots, \mathcal{Z}_{d}$ be the distinct components of $\mathcal{Z}$. We claim that the affine dimension of each component $\mathcal{Z}_{i}$ is at most $t$. If such were not the case for the component $\mathcal{Z}_{i}$, then the intersection defined by (2.3), (2.4) would be improper, and $\mathcal{Z}_{i}$ would belong to the singular locus of $\mathcal{Z}$. Notice that if $1 \leq j \leq u$, then

$$
\frac{\partial}{\partial y_{i}}\left(\sum_{l=1}^{t} \check{y}_{l}^{\kappa_{j}}-\sum_{l=t+1}^{2 t} \check{y}_{l}^{\kappa_{j}}\right)=\kappa_{j} y_{i}^{-1}\left(\sum_{l=1}^{t} \check{y}_{l}^{\kappa_{j}}-\sum_{l=t+1}^{2 t} \check{y}_{l}^{\kappa_{j}}-\omega \check{y}_{i}^{\kappa_{j}}\right)
$$

where $\omega$ is 1 for $1 \leq i \leq t$, and -1 for $t+1 \leq i \leq 2 t$. Thus, when $\mathbf{x}$ satisfies (2.3), (2.4), we find that

$$
\left[\frac{\partial}{\partial y_{i}}\left(\sum_{l=1}^{t} \check{y}_{l}^{\kappa_{j}}-\sum_{l=t+1}^{2 t} \check{y}_{l}^{\kappa_{j}}\right)\right]_{\mathbf{y}=\mathbf{x}}=-\omega \kappa_{j} x_{i}^{-1} \check{x}_{i}^{\kappa_{j}}=-\omega \kappa_{j} x_{i}^{k_{j}-1}\left(x_{1} x_{2} \cdots x_{2 t}\right)^{\kappa_{j}} .
$$

Consequently, by considering the Jacobian determinants arising from the system (2.3), (2.4), and noting that $x_{1} x_{2} \cdots x_{2 t} \neq 0$, we find that the singular locus of $\mathcal{Z}$ is contained within the set of points $\mathbf{x} \in\left(\overline{\mathbb{F}}_{q}^{\times}\right)^{2 t}$ satisfying the simultaneous equations

$$
k_{1} k_{2} \cdots k_{t} \operatorname{det}\left(x_{i_{l}}^{k_{j}-1}\right)_{1 \leq l, j \leq t}=0
$$

with $1 \leq i_{1}<i_{2}<\cdots<i_{t} \leq 2 t$. According to Lemma 2.4(ii), the set of points $\mathbf{x} \in\left(\overline{\mathbb{F}}_{q}^{\times}\right)^{2 t}$ satisfying (2.5) has dimension at most $t-1$, contradicting our earlier hypothesis.

We have shown as before that the components $\mathcal{Z}_{1}, \ldots, \mathcal{Z}_{d}$ of $\mathcal{Z}$ each have dimension at most $t$, and so we may infer from Lemma 2.3 that

$$
M_{t}(q ; \mathbf{k})=\operatorname{card}\left(\mathcal{Z} \cap\left(\mathbb{F}_{q}^{\times}\right)^{2 t}\right) \leq m_{1} m_{2} \cdots m_{t}(q-1)^{t}
$$

This completes the proof of the first estimate of Theorem 1.2.

We next seek to establish the third estimate of Theorem 1.2. On this occasion, we introduce the notational device of writing

$$
\hat{x}_{i}=\prod_{\substack{0 \leq l \leq t \\ l \neq i}} x_{l} \quad(1 \leq i \leq t)
$$

and

$$
\hat{x}_{i}=\prod_{\substack{t+1 \leq l \leq 2 t+1 \\ l \neq i}} x_{l} \quad(t+1 \leq i \leq 2 t) .
$$


We again write $\kappa_{j}$ for $-k_{j}$. Finally, we define $L_{t}(q ; \mathbf{k})$ to be the number of solutions of the system of equations

$$
\begin{aligned}
\prod_{i=0}^{t} x_{i} & =\prod_{i=t+1}^{2 t+1} x_{i}, \\
\sum_{i=1}^{t} \hat{x}_{i}^{\kappa_{j}} & =\sum_{i=t+1}^{2 t} \hat{x}_{i}^{\kappa_{j}} \quad(1 \leq j \leq u), \\
\sum_{i=1}^{t} x_{i}^{k_{j}} & =\sum_{i=t+1}^{2 t} x_{i}^{k_{j}} \quad(u+1 \leq j \leq t),
\end{aligned}
$$

with $\mathbf{x} \in\left(\mathbb{F}_{q}^{\times}\right)^{2 t+2}$. Notice here that in view of the definition of $\hat{x}_{i}$, the degree of the $j$ th equation in (2.7) is $t\left|k_{j}\right|=l_{j}$.

Given a solution $\mathbf{x}$ of the system (1.1) counted by $M_{t}(q ; \mathbf{k})$, one has both $x_{1} \cdots x_{t} \neq 0$ and $x_{t+1} \cdots x_{2 t} \neq 0$. Then given $x_{0} \in \mathbb{F}_{q}^{\times}$, there is a unique element $x_{2 t+1}$ in $\mathbb{F}_{q}^{\times}$for which equation (2.6) holds. Given such a $(2 t+2)$-tuple $\mathbf{x}$, we may multiply the equations of (1.1) with $1 \leq j \leq u$ by the nonzero factor $\left(x_{0} x_{1} \cdots x_{t}\right)^{\kappa_{j}}$ on the left-hand side, and by $\left(x_{t+1} \cdots x_{2 t} x_{2 t+1}\right)^{\kappa_{j}}$ on the right-hand side. In view of relation (2.6), this is the same nonzero factor, and so we obtain the equivalent equations (2.7). In this way we find not only that the system (2.6)-(2.8) is satisfied, but further that

$$
L_{t}(q ; \mathbf{k})=(q-1) M_{t}(q ; \mathbf{k}) .
$$

Recall the definition of the integers $l_{i}(1 \leq i \leq t)$ given in the preamble to the statement of Theorem 1.2. Then keeping in mind the definition of $\hat{x}_{i}$, we find that the system (2.6)-(2.8) is defined by a system of $t+1$ polynomial equations, of respective degrees $t+1$ and $l_{1}, \ldots, l_{t}$, in $2 t+2$ variables. Let $\mathcal{Z}$ be the complete intersection defined by (2.6)-(2.8) with $\mathbf{x} \in\left(\overline{\mathbb{F}}_{q}^{\times}\right)^{2 t+2}$, and let $\mathcal{Z}_{1}, \ldots, \mathcal{Z}_{d}$ be the distinct components of $\mathcal{Z}$. We claim that the affine dimension of each component $\mathcal{Z}_{i}$ is at most $t+1$. If such were not the case for the component $\mathcal{Z}_{i}$, then the intersection defined by (2.6)-(2.8) would be improper, and $\mathcal{Z}_{i}$ would belong to the singular locus of $\mathcal{Z}$. Notice that if $\mathbf{x}$ satisfies (2.6), then

$$
\left[\frac{\partial}{\partial y_{i}}\left(\prod_{l=0}^{t} y_{l}-\prod_{l=t+1}^{2 t+1} y_{l}\right)\right]_{\mathbf{y}=\mathbf{x}}=\omega x_{i}^{-1} \prod_{l=0}^{t} x_{l}
$$

where $\omega$ is 1 for $0 \leq i \leq t$, and -1 for $t+1 \leq i \leq 2 t+1$. Next, when $1 \leq j \leq u$ and $\mathbf{x}$ satisfies (2.6)-(2.8), write

$$
\lambda_{j}(\mathbf{x})=\sum_{l=1}^{t} \hat{x}_{l}^{\kappa_{j}}=\sum_{l=t+1}^{2 t} \hat{x}_{l}^{\kappa_{j}} .
$$


Then, when $1 \leq i \leq t$,

$$
\begin{aligned}
{\left[\frac{\partial}{\partial y_{i}}\left(\sum_{l=1}^{t} \hat{y}_{l}^{\kappa_{j}}-\sum_{l=t+1}^{2 t} \hat{y}_{l}^{\kappa_{j}}\right)\right]_{\mathbf{y}=\mathbf{x}} } & =\left[\kappa_{j} y_{i}^{-1}\left(\sum_{l=1}^{t} \hat{y}_{l}^{\kappa_{j}}-\hat{y}_{i}^{\kappa_{j}}\right)\right]_{\mathbf{y}=\mathbf{x}} \\
& =\kappa_{j} x_{i}^{-1} \lambda_{j}(\mathbf{x})-\kappa_{j} x_{i}^{k_{j}-1}\left(x_{0} x_{1} \cdots x_{t}\right)^{\kappa_{j}} .
\end{aligned}
$$

Likewise, when $t+1 \leq i \leq 2 t$, one finds that

$$
\left[\frac{\partial}{\partial y_{i}}\left(\sum_{l=1}^{t} \hat{y}_{l}^{\kappa_{j}}-\sum_{l=t+1}^{2 t} \hat{y}_{l}^{\kappa_{j}}\right)\right]_{\mathbf{y}=\mathbf{x}}=-\kappa_{j} x_{i}^{-1} \lambda_{j}(\mathbf{x})+\kappa_{j} x_{i}^{k_{j}-1}\left(x_{0} x_{1} \cdots x_{t}\right)^{\kappa_{j}} .
$$

In addition,

$$
\left[\frac{\partial}{\partial y_{0}}\left(\sum_{l=1}^{t} \hat{y}_{l}^{\kappa_{j}}-\sum_{l=t+1}^{2 t} \hat{y}_{l}^{\kappa_{j}}\right)\right]_{\mathbf{y}=\mathbf{x}}=\kappa_{j} x_{0}^{-1} \lambda_{j}(\mathbf{x}) .
$$

We extend the definition of $\lambda_{j}(\mathbf{x})$ by setting $\lambda_{j}(\mathbf{x})=0$ for $u+1<j \leq 2 t$. Then, when $1 \leq i_{1}<i_{2}<\cdots<i_{t} \leq 2 t$, we define the determinant $\Xi(\mathbf{i})=\Xi_{\mathbf{k}}(\mathbf{x} ; \mathbf{i})$ by

$$
\Xi(\mathbf{i})=\operatorname{det}\left(\begin{array}{cc}
x_{0}^{-1} & \mathbf{u}^{T} \\
\mathbf{v} & A
\end{array}\right)
$$

where $\mathbf{u}$ and $\mathbf{v}$ are the column vectors

$$
\mathbf{u}=\left(x_{i_{l}}^{-1}\right)_{1 \leq l \leq t}, \quad \mathbf{v}=\left(x_{0}^{-1} \lambda_{j}(\mathbf{x})\right)_{1 \leq j \leq t},
$$

and

$$
A=\left(-x_{i_{l}}^{k_{j}-1}\left(x_{0} x_{1} \cdots x_{t}\right)^{\kappa_{j}}+x_{i_{l}}^{-1} \lambda_{j}(\mathbf{x})\right)_{1 \leq l, j \leq t} .
$$

Given a singular point $\mathbf{x} \in\left(\overline{\mathbb{F}}_{q}^{\times}\right)^{2 t+2}$ on $\mathcal{Z}$, the determinant $\Xi_{\mathbf{k}}(\mathbf{x} ; \mathbf{i})$ must vanish for $1 \leq i_{1}<i_{2}<\cdots<i_{t} \leq 2 t$. In order to see this, one has only to note that $x_{0} x_{1} \cdots x_{t}=$ $x_{t+1} \cdots x_{2 t} x_{2 t+1} \neq 0$, and to observe that the Jacobian determinant, corresponding to the partial derivatives indexed by $x_{0}, x_{i_{1}}, \ldots, x_{i_{t}}$, vanishes if and only if $\Xi(\mathbf{i})=0$. For $1 \leq l \leq t$, we may subtract the first column multiplied by $x_{0} x_{i_{l}}^{-1}$ from the $(l+1)$ th column. In this way we find that

$$
\Xi(\mathbf{i})=\operatorname{det}\left(\begin{array}{cc}
x_{0}^{-1} & O \\
\mathbf{v} & B
\end{array}\right)
$$

where

$$
B=\left(-x_{i_{l}}^{k_{j}-1}\left(x_{0} x_{1} \cdots x_{t}\right)^{\kappa_{j}}\right)_{1 \leq l, j \leq t} .
$$

But then $\Xi(\mathbf{i})=x_{0}^{-1} \operatorname{det}(B)$, and hence $\Xi(\mathbf{i})$ vanishes if and only if

$$
\operatorname{det}\left(x_{i_{l}}^{k_{j}-1}\right)_{1 \leq l, j \leq t}=0
$$


From the above discussion, we find that if $\mathcal{Z}_{i}$ has dimension exceeding $t+1$, then the set of points $\left(x_{1}, x_{2}, \ldots, x_{2 t}\right) \in\left(\overline{\mathbb{F}}_{q}^{\times}\right)^{2 t}$ for which $\left(x_{0}, x_{1}, \ldots, x_{2 t+1}\right)$ lies on $\mathcal{Z}_{i}$ must be contained in $\mathfrak{Y}_{2 t}(\mathbf{k})$. From Lemma 2.4(ii), this set has dimension at most $t-1$. But the equation (2.6) ensures that $x_{0}$ is uniquely determined from $x_{2 t+1}$ together with a given choice of $\left(x_{1}, x_{2}, \ldots, x_{2 t}\right)$, and so $\mathcal{Z}_{i}$ can have dimension at most $(t-1)+1=t$. This contradicts our earlier hypothesis that $\operatorname{dim}\left(\mathcal{Z}_{i}\right)>t+1$.

We have shown on this occasion that the components $\mathcal{Z}_{1}, \ldots, \mathcal{Z}_{d}$ each have dimension at most $t+1$, so by Lemma 2.3 we deduce that

$$
L_{t}(q ; \mathbf{k})=\operatorname{card}\left(\mathcal{Z} \cap\left(\mathbb{F}_{q}^{\times}\right)^{2 t+2}\right) \leq(t+1) l_{1} l_{2} \cdots l_{t}(q-1)^{t+1} .
$$

Consequently, relation (2.9) delivers the estimate

$$
M_{t}(q ; \mathbf{k}) \leq(t+1) l_{1} l_{2} \cdots l_{t}(q-1)^{t},
$$

and this confirms the third estimate of Theorem 1.2.

An alternative approach is required to establish the second estimate of Theorem 1.2. Given a solution $\mathbf{x} \in\left(\mathbb{F}_{q}^{\times}\right)^{2 t}$ of the system (1.1), there is a unique element $\mathbf{y} \in\left(\mathbb{F}_{q}^{\times}\right)^{2 t}$ for which $x_{i} y_{i}=1(1 \leq i \leq 2 t)$. Consequently, if we define $K_{t}(q ; \mathbf{k})$ to be the number of solutions of the system of equations

$$
\begin{array}{rlrl}
\sum_{i=1}^{t}\left(y_{i}^{\kappa_{j}}-y_{t+i}^{\kappa_{j}}\right)=0 & & (1 \leq j \leq u), \\
\sum_{i=1}^{t}\left(x_{i}^{k_{j}}-x_{t+i}^{k_{j}}\right)=0 & & (u+1 \leq j \leq t), \\
x_{l} y_{l}=1 & (1 \leq l \leq 2 t),
\end{array}
$$

with $\mathbf{x}, \mathbf{y} \in\left(\mathbb{F}_{q}^{\times}\right)^{2 t}$, then one finds that

$$
K_{t}(q ; \mathbf{k})=M_{t}(q ; \mathbf{k}) .
$$

Let $\mathcal{Z}$ be the complete intersection defined by the system (2.10)-(2.12) with $\mathbf{x}, \mathbf{y} \in\left(\mathbb{F}_{q}^{\times}\right)^{2 t}$. Let $\mathcal{Z}_{1}, \ldots, \mathcal{Z}_{d}$ be the distinct components of $\mathcal{Z}$. We claim that the affine dimension of each component $\mathcal{Z}_{i}$ is at most $t$. If such were not the case, then the intersection defined by (2.10)-(2.12) would be improper, and $\mathcal{Z}_{i}$ would belong to the singular locus of $\mathcal{Z}$. The Jacobian determinants corresponding to the system (2.10)-(2.12) are not particularly simple to describe, and so we must introduce some additional notation. The system (2.10)-(2.12) possesses $3 t$ equations and $4 t$ variables. Let $h_{1}, \ldots, h_{t}$ be integers with $1 \leq h_{1}<h_{2}<\cdots<h_{t} \leq 2 t$, and write $\mathcal{H}=\left\{h_{1}, \ldots, h_{t}\right\}$ and define $\mathcal{I}=\mathcal{I}(\mathcal{H})$ by $\mathcal{I}=\{1, \ldots, 2 t\} \backslash \mathcal{H}$. Then $\mathcal{I}$ is a set of integers $i_{1}, \ldots, i_{t}$ with $1 \leq i_{1}<\cdots<i_{t} \leq 2 t$. For each set $\mathcal{H}$ of the above type, we define the Jacobian determinant $\Upsilon(\mathcal{H})=\Upsilon_{\mathbf{k}}(\mathbf{x} ; \mathcal{H})$ by

$$
\Upsilon(\mathcal{H})=\operatorname{det}\left(\begin{array}{lll}
A & O & B \\
O & C & O \\
O & O & F \\
D & E & O
\end{array}\right),
$$


where $A, B, C$ are the diagonal matrices

$$
A=\operatorname{diag}\left(y_{h}\right)_{h \in \mathcal{H}}, \quad B=\operatorname{diag}\left(x_{h}\right)_{h \in \mathcal{H}}, \quad C=\operatorname{diag}\left(y_{i}\right)_{i \in \mathcal{I}(\mathcal{H})},
$$

and $D, E, F$ are the generalized Vandermonde matrices

$$
D=\left(k_{j} x_{h_{l}-1}^{k_{j}-1}\right) \underset{\substack{1 \leq l \leq t \\ u+1 \leq j \leq t}}{ }, \quad E=\left(k_{j} x_{i_{l}-1}^{k_{j}-1}\right)_{\substack{1 \leq l \leq t \\ u+1 \leq j \leq t}}, \quad F=\left(\kappa_{j} y_{h_{l}-1}^{\kappa_{j}-1}\right)_{\substack{1 \leq l \leq t \\ 1 \leq j \leq u}} .
$$

That $\Upsilon(\mathcal{H})$ is indeed a Jacobian determinant may be seen by rearranging the equations comprising (2.10)-(2.12) to correspond to the rows of $\Upsilon(\mathcal{H})$, so that the first $t$ equations become $x_{h} y_{h}=1(h \in \mathcal{H})$, the next $t$ become $x_{i} y_{i}=1(i \in \mathcal{I}(\mathcal{H}))$, and the final $t$ become the $t$ equations of (2.10) and (2.11). Likewise, we rearrange the partial derivatives so that the first $t$ columns of $\Upsilon(\mathcal{H})$ correspond to the partial derivatives $\partial / \partial x_{h}(h \in \mathcal{H})$, the second $t$ correspond to the partial derivatives $\partial / \partial x_{i}(i \in \mathcal{I}(\mathcal{H}))$, and the third $t$ correspond to the partial derivatives $\partial / \partial y_{h}(h \in \mathcal{H})$. It follows, in particular, that if $\mathcal{Z}_{i}$ has dimension exceeding $t$, then its points satisfy the system of equations $x_{i} y_{i}=1(1 \leq i \leq 2 t)$, and

$$
\Upsilon(\mathcal{H})=0 \quad\left(\mathcal{H}=\left\{h_{1}, \ldots, h_{t}\right\} \subset\{1,2, \ldots, 2 t\}\right) .
$$

One has

$$
\operatorname{det}(\Upsilon(\mathcal{H}))=\operatorname{det}(C) \operatorname{det}\left(\begin{array}{cc}
A & B \\
O & F \\
D & O
\end{array}\right)
$$

For $1 \leq l \leq t$, we may subtract $x_{h_{l}} y_{h_{l}}^{-1}$ times the $l$ th column of the last determinant from the $(t+l)$ th column, without affecting its value. In this way, we find that

$$
\operatorname{det}(\Upsilon(\mathcal{H}))=\operatorname{det}(C) \operatorname{det}\left(\begin{array}{cc}
A & O \\
O & F \\
D & G
\end{array}\right)
$$

where

$$
G=\left(k_{j} x_{h_{l}}^{k_{j}} y_{h_{l}}^{-1}\right) \underset{\substack{1 \leq l \leq t \\ u+1 \leq j \leq t}}{ } .
$$

Making use of the relations $x_{i} y_{i}=1(1 \leq i \leq 2 t)$, we find that

$$
\begin{aligned}
\operatorname{det}(\Upsilon(\mathcal{H})) & = \pm \operatorname{det}(A) \operatorname{det}(C) \operatorname{det}\left(k_{j} x_{h_{l}}^{k_{j}} y_{h_{l}}^{-1}\right)_{1 \leq l, j \leq t} \\
& = \pm k_{1} \cdots k_{t}\left(\prod_{h \in \mathcal{H}} x_{h}\right)\left(\prod_{i \in \mathcal{I}(\mathcal{H})} y_{i}\right) \operatorname{det}\left(x_{h_{l}}^{k_{j}-1}\right)_{1 \leq l, j \leq t} .
\end{aligned}
$$

From the above discussion, we find that if $\mathcal{Z}_{i}$ has dimension exceeding $t$, then the set of points $\mathbf{x} \in\left(\overline{\mathbb{F}}_{q}^{\times}\right)^{2 t}$ for which $(\mathbf{x}, \mathbf{y})$ lies on $\mathcal{Z}_{i}$ must be contained in $\mathfrak{Y}_{2 t}(\mathbf{k})$. But Lemma 2.4(ii) shows that the latter has dimension at most $t-1$. Since for $(\mathbf{x}, \mathbf{y}) \in \mathcal{Z}_{i}$ 
one has $x_{i} y_{i}=1 \quad(1 \leq i \leq 2 t)$, each coordinate $y_{i}$ is uniquely determined from $x_{i}$, whence $\mathcal{Z}_{i}$ itself can have dimension at most $t-1$, contradicting our earlier hypothesis.

The components $\mathcal{Z}_{1}, \ldots, \mathcal{Z}_{d}$ of $\mathcal{Z}$ therefore each have dimension at most $t$, whence by Lemma 2.3 we obtain the estimate

$$
K_{t}(q ; \mathbf{k})=\operatorname{card}\left(\mathcal{Z} \cap\left(\mathbb{F}_{q}^{\times}\right)^{4 t}\right) \leq 2^{2 t} \kappa_{1} \cdots \kappa_{u} k_{u+1} \cdots k_{t}(q-1)^{t} .
$$

In view of (2.13), the second estimate for $M_{t}(q ; \mathbf{k})$ asserted by Theorem 1.2 follows on recalling that $\kappa_{i}=-k_{i}$. This completes our account of the proof of Theorem 1.2.

The conclusions of Corollary 1.3 follow at once from Theorems 1.1 and 1.2 by means of the argument of Theorem 1.2 of Cochrane and Pinner [6]. Under the hypotheses of Corollary 1.3, the estimate of Cochrane and Pinner shows that

$$
|S(\chi, f)|<(p-1)^{1-2 / t} p^{1 /(2 t)} M^{1 / t^{2}},
$$

where $M=N_{t}(p ; \mathbf{k})$ when $k_{i}>0(1 \leq i \leq t)$, and otherwise $M=M_{t}(p ; \mathbf{k})$. In the first instance, Theorem 1.1 delivers the bound

$$
|S(\chi, f)|<p^{1-3 /(2 t)}\left(k_{1} k_{2} \cdots k_{t} p^{t}\right)^{1 / t^{2}}=\left(k_{1} \cdots k_{t}\right)^{1 / t^{2}} p^{1-1 /(2 t)} .
$$

If the exponents $k_{i}$ are not all positive, then one obtains in like manner the remaining estimates of Corollary 1.3 as immediate corollaries of Theorem 1.2.

\section{Acknowledgement}

The author is grateful to the referee for useful comments.

\section{References}

[1] N. M. Akulinchev, 'Bounds for rational trigonometric sums of a special type', Dokl. Akad. Nauk SSSR 161 (1965), 743-745.

[2] J. Bourgain, 'Mordell's exponential sum estimate revisited', J. Amer. Math. Soc. 18 (2005), $477-499$.

[3] A. Cafure and G. Matera, 'Improved explicit estimates on the number of solutions of equations over a finite field', Finite Fields Appl. 12 (2006), 155-185.

[4] T. Cochrane, J. Coffelt and C. Pinner, 'A further refinement of Mordell's bound on exponential sums', Acta Arith. 116 (2005), 35-41.

[5] T. Cochrane, J. Coffelt and C. Pinner, 'A system of simultaneous congruences arising from trinomial exponential sums', J. Théor. Nombres Bordeaux 18 (2006), 59-72.

[6] T. Cochrane and C. Pinner, 'An improved Mordell type bound for exponential sums', Proc. Amer. Math. Soc. 133 (2005), 313-320.

[7] T. Cochrane, C. Pinner and J. Rosenhouse, 'Bounds on exponential sums and the polynomial Waring problem mod p', J. Lond. Math. Soc. (2) 67 (2003), 319-336.

[8] R. Hartshorne, Algebraic Geometry (Springer, Berlin, 1977).

[9] J. Heintz and C.-P. Schnorr, 'Testing polynomials which are easy to compute', in: Logic and Algorithmic (Zurich, 1980), Monographies de l'Enseignement Mathématique, 30 (Univ. Genève, Geneva, 1982), pp. 237-254. 
[10] A. A. Karatsuba, 'Estimates of complete trigonometric sums', Mat. Zametki 1 (1967), 199-208.

[11] L. J. Mordell, 'On a sum analogous to Gauss's sum', Q. J. Math. Oxford 3 (1932), 161-167.

[12] I. M. Vinogradov, 'New estimates for Weyl sums', Dokl. Akad. Nauk SSSR 8 (1935), 195-198.

[13] A. Weil, 'On some exponential sums', Proc. Natl. Acad. Sci. USA 34 (1948), 204-207.

[14] T. D. Wooley, 'On simultaneous additive equations, III', Mathematika 37 (1990), 85-96.

[15] T. D. Wooley, 'A note on simultaneous congruences', J. Number Theory 58 (1996), 288-297.

[16] H. B. Yu, 'Estimates for complete exponential sums of special types', Math. Proc. Cambridge Philos. Soc. 131 (2001), 321-326.

TREVOR D. WOOLEY, School of Mathematics, University of Bristol, University Walk, Clifton, Bristol BS8 1TW, UK

e-mail: matdw@bristol.ac.uk, Trevor.Wooley@bristol.ac.uk 